\title{
Stereo-based Pedestrian Detection in Crosswalks for Pedestrian Behavioural Modelling Assessment
}

\author{
D. F. Llorca ${ }^{1}$, I. Parra ${ }^{2}$, R. Quintero ${ }^{1}$, C. Fernández ${ }^{1}$, R. Izquierdo ${ }^{1}$ and M. A. Sotelo ${ }^{1}$ \\ ${ }^{1}$ Computer Engineering Department, University of Alcalá, Alcalá de Henares, Madrid, Spain \\ ${ }^{2}$ Department of Signal Theory and Communications, Polytechnic University of Madrid, Madrid, Spain \\ llorca@aut.uah.es
}

Keywords: $\quad$ Stereo-vision, Pedestrian Detection, Pedestrian Behaviour, Pedestrian Crossings, Accepted Gap.

\begin{abstract}
In this paper, a stereo- and infrastructure-based pedestrian detection system is presented to deal with infrastructure-based pedestrian safety measurements as well as to assess pedestrian behaviour modelling methods. Pedestrian detection is performed by region growing over temporal 3D density maps, which are obtained by means of stereo reconstruction and background modelling. 3D tracking allows to correlate the pedestrian position with the different pedestrian crossing regions (waiting and crossing areas). As an example of an infrastructure safety system, a blinking luminous traffic sign is switched on to warn the drivers about the presence of pedestrians in the waiting and the crossing regions. The detection system provides accurate results even for nighttime conditions: an overall detection rate of $97.43 \%$ with one false alarm per each 10 minutes. In addition, the proposed approach is validated for being used in pedestrian behaviour modelling, applying logistic regression to model the probability of a pedestrian to cross or wait. Some of the predictor variables are automatically obtained by using the pedestrian detection system. Other variables are still needed to be labelled using manual supervision. A sequential feature selection method showed that time-to-collision and pedestrian waiting time (both variables automatically collected) are the most significant parameters when predicting the pedestrian intent. An overall predictive accuracy of $93.10 \%$ is obtained, which clearly validates the proposed methodology.
\end{abstract}

\section{INTRODUCTION}

The European Commission's goal of reducing road fatalities by $50 \%$ in the period $2000-2010$ was almost achieved (actual reduction is estimated to be $44 \%$ (EC, 2012)) and the same target has been adopted in the period $2010-2020$. Within this context, pedestrians - including people with disabilities or reduced mobility and orientation - account for the $20 \%$ of the fatalities in EU-24 with more than 6.000 pedestrians died in road traffic accidents in 2010. The $75 \%$ of pedestrians fatalities takes place in urban environments and around the $25 \%$ occurs in on or close to a pedestrian crossing (EC, 2012). Accordingly, new solutions specifically devised to increase the safety of pedestrians at urban crosswalks can be of great help to reach this ambitious mid-term goal.

Improvements in pedestrian safety at crosswalks can be addressed from two perspectives: vehicle and/or infrastructure. In both cases, the systems have to be devised to allow reliable detection and tracking of pedestrians and other road users. However, although robust detection and tracking (Parra et al., 2007) are essential pre-requisites for the successful development of these systems, they are not sufficient. Hence, besides locating, recognising and tracking the pedestrians, the most relevant parameters by which the intention of the pedestrians to cross or wait can be unambiguously predicted have to be identified (Schmidt and Farber, 2009). Thus, accurate pedestrian path prediction and action recognition can be possible from both the vehicle (Keller and Gavrila, 2014), (Quintero et al., 2014), and the infrastructure (Kohler et al., 2013), allowing the development of safety measures.

This paper presents the first stage of an infrastructure-based pedestrian prediction system devised to increase the safety of pedestrians as well as to facilitate the analysis of the main parameters involved in pedestrians behaviour at crosswalks in naturalistic traffic conditions. A wide-angle stereo-based pedestrian detection and tracking system has been developed to be used in both daytime and nighttime conditions. The different areas of the crosswalk are 
previously defined (sidewalks, curbs, and crossing area). Thus, the system can deal with automatic estimation of some of the main parameters with influence in pedestrians intent (e.g., waiting time, pedestrian speed, path changes, number of pedestrians, relative pedestrian-to-vehicle time-to-collision, acceptance gaps, etc.). Although some parameters are still needed to be obtained by manual supervision (number of observations at curb, number of observations while crossing, approximated age, gender, etc.) of video sequences, the proposed approach drastically reduces the time dedicated to this task. Performance results are provided from three different crosswalks corresponding to the University Campus of the University of Alcalá (Alcalá de Henares, Madrid, Spain). In addition, the obtained pedestrian behavioural parameters are analysed to validate the proposed approach for pedestrian behaviour modelling.

The rest of the paper is organised as follows: Section 2 describes the state-of-the-art concerning stereoand infrastructure-based approaches for pedestrian detection as well as related work concerned with pedestrian road crossing behaviour. The stereo-based pedestrian detection and tracking system is presented in Section 3. In Section 4 an overview of the main parameters surveyed and the data collection process are presented. Experimental results are described in Section 5. Conclusions and future works are finally addressed in Section 6.

\section{RELATED WORK}

Stereo-based pedestrian detection is a well-known topic in the context of Advanced Driver Assistance Systems (ADAS) and Intelligent Transportation Systems (ITS). Vehicle-based pedestrian protection systems have been recently surveyed in (Gerónimo and López, 2014). The use of stereo vision has taken a key role in this context since it enhances both the region of interest selection stage (Llorca et al., 2012) and the classification performance (Keller et al., 2011), providing depth measurements that are essential for collision avoidance manoeuvres such as emergency braking (Milanes et al., 2012) or automatic steering (Llorca et al., 2011). In the context of infrastructurebased pedestrian detection, the use of monocular approaches has been widely proposed since background subtraction (Álvarez et al., 2014) or motion history techniques (Kohler et al., 2013) can be directly applicable. However, accurate depth cues are still needed to allow the applicability of infrastructurebased safety measurements including V2I communications, traffic lights control, etc. Thus, in (Weimer et al., 2011) a multisensor platform is used to detect pedestrians at intersections, including laser scanning systems and far-infrared (FIR) cameras. We also remark the well-known SafeWalk commercial system (Favoreel, 2011) which is the first stereo-based pedestrian detection platform available for its use at urban intersections. The main drawbacks of this system are its narrow field of view and its close range, which limits its use to the pedestrian waiting area at sidewalks. Accordingly, monitoring a multiple lane crosswalk including the pedestrian waiting areas requires at least two SafeWalk systems for the waiting areas and one C-Walk (monocular) for the crosswalk. Thus, stereo measurements are only available at waiting zones. In this paper we propose the use of a wide-angle stereobased pedestrian system able to monitor a two-lane crosswalk including pedestrian waiting areas.

Considering pedestrian behaviour modelling, most of the approaches are mainly based on quite standardised methodologies such as the use of questionnaires, personal interviews and statistical analysis of traditional crash data. The use of infrastructurebased surveillance data from high resolution cameras or radar sensors, using actors (Schmidt and Farber, 2009) or naturalistic data (Kadali and Vedagiri, 2013) has been recently proposed allowing the connection of new relevant variables with the pedestrian's intention to cross in the short time. Some examples are: vehicle-to-pedestrian distance and time-to-collision, speed, pedestrian gaze frequency and duration before and while crossing, number of crossing attempts, accepted gap, etc. It is worth to mention that surveillance data is evaluated in a fully manual fashion, which clearly limits their applicability and standardisation potentials. In this paper we propose to automatically assess some of these variables by means of the stereo-based pedestrian monitoring system. Variables such as time spent at the curb, speed of the pedestrian while crossing the road, pedestrian speed and path changes, number of pedestrians in the group, etc., can be automatically collected.

\section{PEDESTRIAN MMONITORING}

\subsection{System Architecture}

The stereo platform is composed of two CMOS cameras with optics of $2.8 \mathrm{~mm}$ (wide-angle) and a baseline of $30 \mathrm{~cm}$, integrated in a platform that includes two cameras housing and an IR illuminator directly controlled by a photocell (see Figure 1). Cameras are connected by FireWire to an industrial PC. A specific HW has been devised to control the external trigger 


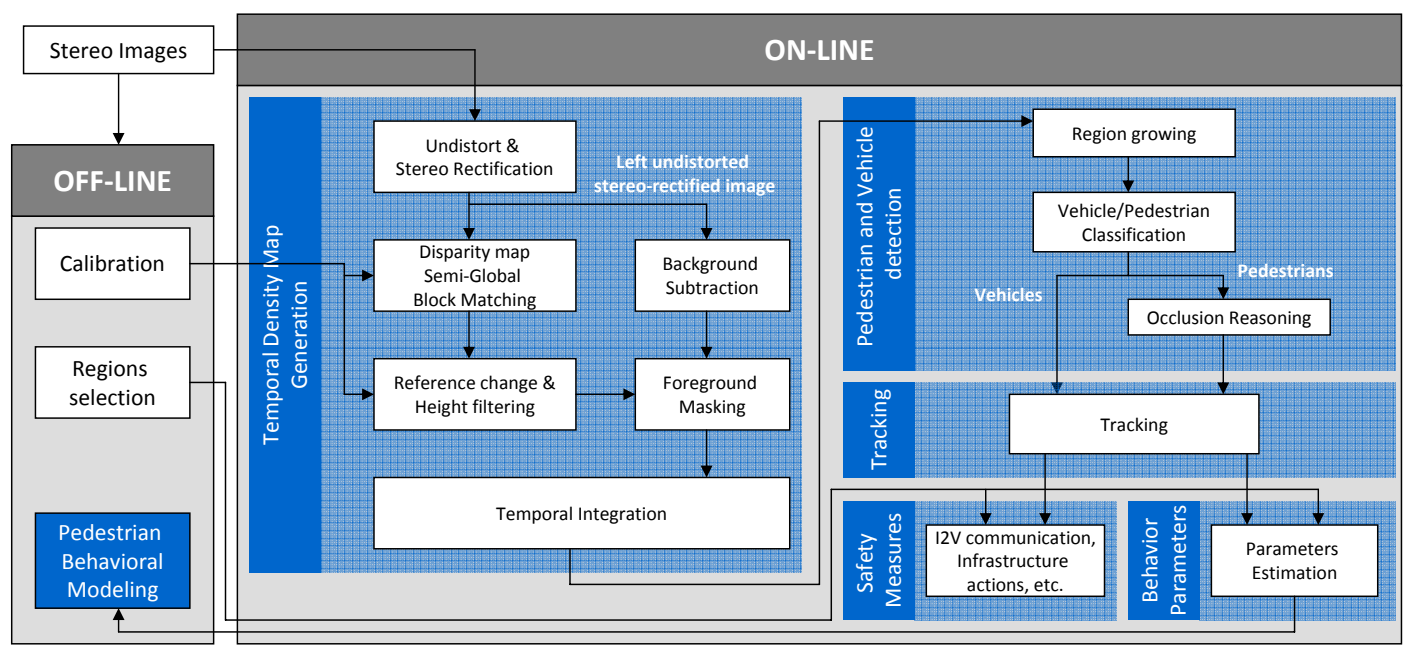

Figure 3: System overview: off-line and on-line tasks.

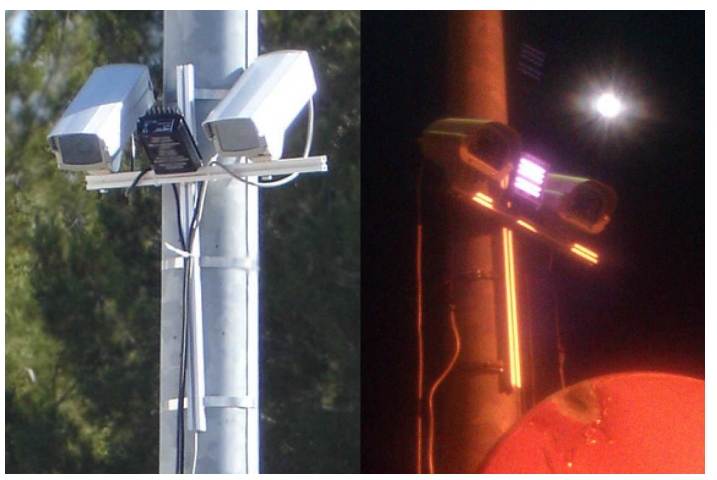

Figure 1: Stereo platform with IR illuminator.

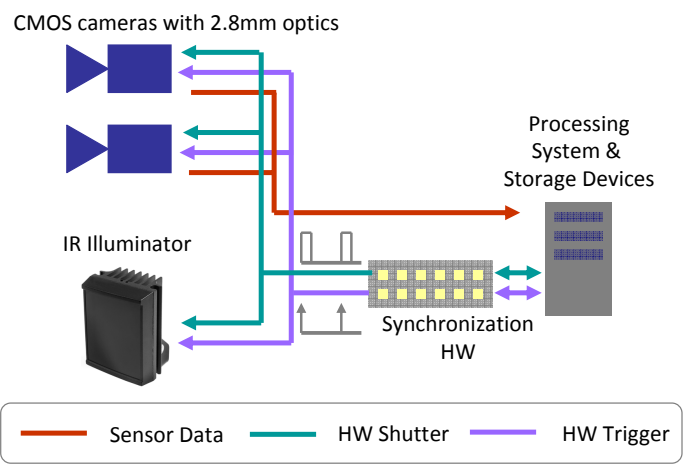

Figure 2: Sensor architecture.

of the cameras and the IR illuminator, including a serial communication with the PC. When the photocell is activated, the IR illuminator is pulsed with a work cycle defined by the specific shutter time of both cameras. Note that camera settings such as gain and shutter are automatically adapted to the illumination conditions. Figures 2 and 3 depict the general architecture of the system.

\subsection{Stereo-based Pedestrian Detection}

The stereo system has to be calibrated first. Since the platform is expected to be installed once and considered fixed since then, we only perform one calibration including intrinsic camera parameters, extrinsic relationship of the stereo rig, and extrinsic relationship between the left camera and the ground plane. Both intrinsic and extrinsic parameters are obtained by means of standard chessboard calibration procedure (see Figure 4. A specific calibration software that makes use of calibration functions of OpenCV library has been developed to automatically detect chessboard corners from each pair of images, and provide calibration parameters of the stereo rig.

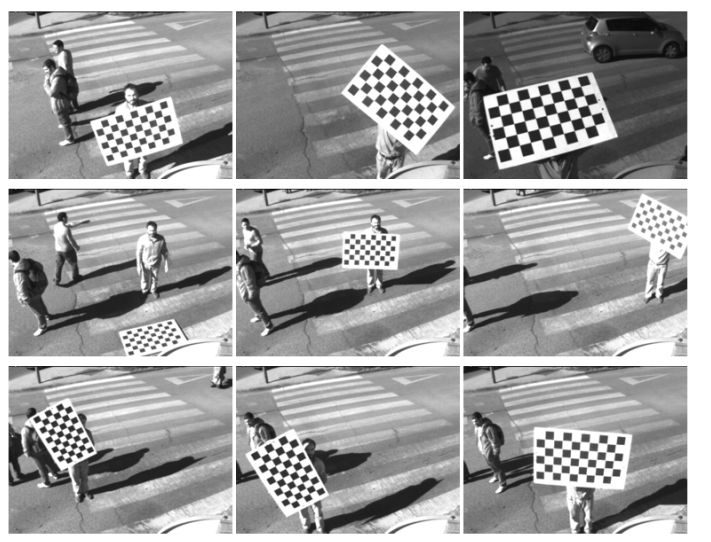

Figure 4: Calibration images used for computing both intrinsic and extrinsic stereo rig parameters.

The images of the camera are firstly undistorted and then stereo-rectified to assure that a point on the left image produces an horizontal epipolar line at the same $v$-coordinate. Then, disparities are computed 
using OpenCV's semi-global block matching algorithm. The 3D-reconstruction of the scene is thus referenced to the left camera optical centre. However, in order to use 3D-measurements in a consistent fashion to assign the pedestrian position to the different regions (waiting regions and crossing area), a rigid transformation between the stereo platform and the ground plane has to be computed. A supervised procedure has been designed to that effect. First, the translation vector that includes the height of the camera, and the displacement between the pole and the crosswalk is manually obtained. Then rotation angles are adjusted using a manual procedure in which the operator has to rotate the 3D-map obtained from the scene to fit the ground plane with a vertical plane using a bird's eye view representation. The first step consists in rotating over the $Y$-axis to assure that $Z$ axis gets parallel to the crosswalk. Thus the yaw angle is obtained. Then a $90^{\circ}$ rotation over the $Z$-axis is performed including the adjustment needed to minimise the width of the obtained plane which represents the roll angle. Finally, pitch angle is estimated by rotating over the $X$-axis until the obtained plane appears in a vertical position. An example of the procedure is presented in Figure 5.

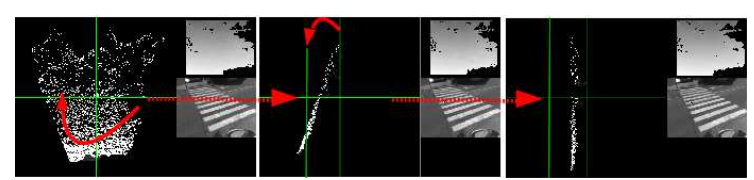

Figure 5: Manual procedure to obtain the rotation angles. Left: original bird's eye view and rotation over the $Z$-axis. Middle: pitch correction by rotating over the $X$-axis. Right: final result.

The last step corresponding to the manual installation process consists in defining the pedestrian waiting regions and the crossing area. The regions are selected using the undistorted left image. The selected image points are considered as pertaining to the ground plane ( $Y$-coordinate or height is equal to zero). Thus, the other two components of the 3D position (lateral position and depth or distance) can be easily obtained. The selected regions are then translated into the 3D-map and they will be used to classify the 3D-position of the detected and tracked pedestrians.

Pedestrian detection is performed by using a density map or bird's-eye map. The 3D-points are projected on a $X Z$-map (road plane). Considering the road as flat and taking into account that $3 \mathrm{D}$-points are related to the road plane, a 3D filtering procedure is applied to remove points inside the range $0.2 m<$ $Y<2 m$. Thus points pertaining to the ground plane and to very high regions are not considered for pedes- trian detection. As described in (Nedevschi et al., 2009), the density map can be seen as an accumulator buffer. Each projected point within the previously defined height margins (after 3D-filtering) adds a value to the accumulation buffer. A pixel in the accumulator buffer covers a small area (around $80 \times 80 \mathrm{~mm}$ ). The weights that each point adds to the density map follow a Gaussian distribution with the maximum at the centre pixel and decreasing in the neighbouring pixels. Because the influence of each 3D-point on the density map is cumulative, the resulting map will contain large values in regions with a high density of $3 \mathrm{D}$ points. In order to reduce the effect of stereo matching errors, a temporal density map is finally obtained by integrating the projected points during the last three frames (around $200 \mathrm{msec}$ at $15 \mathrm{~Hz}$ ). An example of this temporal density map is depicted in Figure 6. As can be observed, the obtained map includes information related with pedestrians and other vertical objects such as poles, trees, etc. In order to remove static objects a dynamical background subtraction algorithm -proposed by the authors in (Álvarez et al., 2012) for monocular traffic detection- is applied to mask the disparity map with the foreground objects. The background is adapted with a learning rate of 0.1 minimising the probabilities of incorporating pedestrians or vehicles inside the background model.
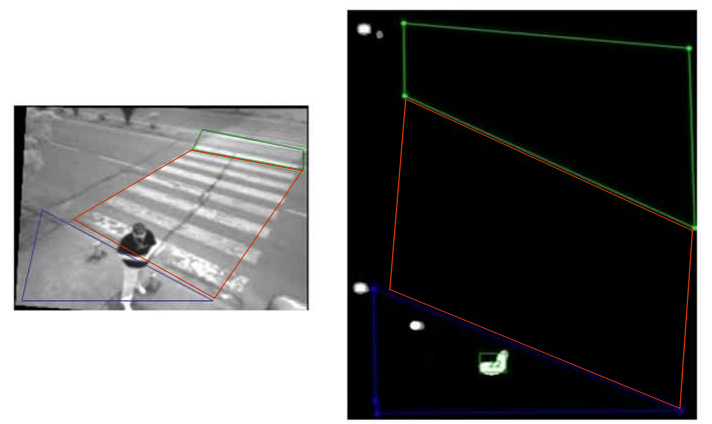

Figure 6: Left: undistorted left image with selected regions. Right: temporal density map of filtered points.

Object segmentation is performed on the filteredtemporal density map (masking with background subtraction result and removing ground plane and very high 3D-points), using a region-growing algorithm. The result of the segmentation is a list of object hypotheses on the density map. Each candidate is firstly classified as pedestrian or vehicle analysing their velocities (orientation and speed), size and the image location of the first appearance in the scene. Some restrictions over the minimum and maximum blobs size are applied to filter small objects and split big objects classified as pedestrians. The shape of each object with a size that may correspond to multiple pedestrians is analysed using the occlusion reasoning algo- 
Table 1: Collected variables to model pedestrian behaviour.

\begin{tabular}{llll}
\hline Variable & Type & $\begin{array}{l}\text { Manual / } \\
\text { Automatic }\end{array}$ & Description \\
\hline Time-to-collision & Cont. & M/A & Gap time when pedestrian decides to wait or cross \\
Distance & Cont. & M/A & Gap distance when pedestrian decides to wait or cross \\
Vehicle Speed & Cont. & M/A & Speed of the vehicle at crosswalk area \\
Waiting time & Cont. & A & Duration of time spent by a pedestrian for accepting gap \\
Pedestrian Speed & Cont. & A & Speed of pedestrian while crossing \\
Curb observ. time & Cont. & M & Duration of pedestrian observing time for accepting gap \\
Observations curb & Cont. & M & Number of observations made by a pedestrian at the curb \\
Gender & Cat. & M & Male/Female \\
Age & Cat. & M & Elders/Middle/Child \\
Platoon & Cat. & A & Number of pedestrians in the group (1, 2 or $>2)$ \\
Baggage & Cat. & M & Whether pedestrian is carrying baggage or not \\
Type of vehicle & Cat. & M/A & Heavy/Car/Powered Two Wheeler \\
Gap acceptance & Cat. & A & Whether pedestrian at the curb accepts/rejects the gap \\
\hline * Cont. $=$ Continuous; Cat. $=$ Categorical; M $=$ Manual; A = Automatic.
\end{tabular}

rithm presented in (Álvarez et al., 2014) which makes use of the compactness, the convexity and the convex hull to divide the blob in multiple blobs (note that this procedure is only applied to object hypotheses previously selected as vehicles).

Tracking is carried out using a linear Kalman filter. The motion of both pedestrians and vehicles are modelled using a constant velocity model, allowing accelerations by means of process noise. The state variables are pixel and 3D positions and their corresponding velocities. Only the pixel and the 3D positions are considered in the measurement vector. Data association problem is carried out by means of 3D Mahalanobis distance, template matching (normalised cross-correlation) and Hungarian assignment as in (Parra et al., 2007).

\section{PEDESTRIAN BEHAVIOUR PARAMETERS}

Considering pedestrians road crossing behaviour modelling, the use of surveillance data has been essential to obtain relevant information. Gap acceptance theory played a key role in these studies. Deterministic approaches are mainly based on the computation of a critical gap that assumes that all pedestrian intent are homogeneous and consistent (Das et al., 2005). However, in other studies it has been concluded that gap acceptance behaviour depends on many factors (Kadali and Vedagiri, 2013), (Koh and Wong, 2014), (Schmidt and Farber, 2009) leading to the use of probabilistic approaches. This is a more realistic approach mainly based on the assumption that pedestrian's minimum gap acceptance is a random variable. The probabilistic methods, which can be generative or discriminative, incorporate intrinsic variables (age, gender, etc.) and traffic attributes (time-to-collision, relative distance, type of vehicle, etc.) in order to estimate the probability of a pedestrian to cross or wait. This probability has to be consistent with the aforementioned variables.

In previous approaches all variables are manually obtained by tedious manual labelling procedures applied on the surveillance data. However, the stereobased pedestrian detection module can be here applied to automatically obtain some of these variables. Following similar approaches of previous studies, we have selected some important variables that are listed in Table 1. A brief description and some properties of each variable are depicted in Table 1 . The variables can be continuous or discrete (categorical), and can be manually or automatically selected. The manual procedure is based on visual analysis of the video sequences, which were recorded at $30 \mathrm{~Hz}$. Accordingly, the accuracy of the temporal measurements can not be better than $33 \mathrm{msec}$. When vehicles are inside the field of view of the stereovision system at the time when the pedestrian is located at the curb, time-to-collision, relative distance and vehicle speed measurements are directly obtained using stereo data. However, when vehicles are out of the stereo field of view, these variables are estimated using the difference of the time when the vehicle passed the position where pedestrian stands assuming a constant velocity model. To overcome this limitation other sensors would have to be included to increase the range and the field of view. Table 1 summarises all the variables obtained to deal with pedestrian behaviour modelling.

\section{EXPERIMENTS}

\subsection{Pedestrian Detection Results}

The proposed infrastructure- and stereo-based pedes- 
Table 2: Datasets global description.

\begin{tabular}{lccc}
\hline Id. & $\begin{array}{c}\text { Lighting } \\
\text { Conditions }\end{array}$ & $\begin{array}{c}\text { Duration } \\
\text { (minutes) }\end{array}$ & $\begin{array}{c}\text { Pedestrians } \\
\text { Intent }\end{array}$ \\
\hline 1 & Daytime & 4,1 & 30 \\
2 & Daytime & 9,4 & 159 \\
3 & Nighttime & 15,7 & 70 \\
\hline Total & & 29,28 & 259 \\
\hline
\end{tabular}

trian detection system is tested in three different twolane unsignalised crosswalks. As can be seen in Figure 7 the system is installed and configured to cover the two-lane crossing region and two pedestrian waiting areas. Table 2 summarises the duration (at $30 \mathrm{~Hz}$ ), lighting conditions and number of pedestrians intent collected at the three locations.

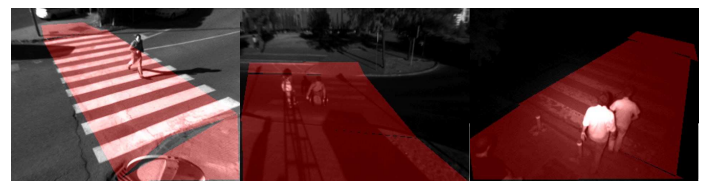

Figure 7: Three different locations covering the two-lane crossing region and two pedestrian waiting areas, including different lighting conditions.

Considering the global architecture of the system presented in Figure 3 we have devised a safety system that includes a luminous intermittent traffic signal that is switched on when pedestrians are waiting or crossing to warn the driver. Accordingly, we define the following variables:

- True Positives (TP): warning is switched on and pedestrians are waiting or crossing

- True Negatives (TN): warning is switched off and no pedestrians are waiting or crossing

- False Positives (FP): warning is switched on and no pedestrians are waiting or crossing

- False Negatives $(F N)$ : warning is switched off and pedestrians are waiting or crossing

Detection rate (DR) is defined as $D R=T P /(T P+$ $F N)$. Taking into account the previous definitions, Table 3 depicts the obtained results. As can be observed the system provides very accurate results even for nighttime conditions. The number of false positives remains very low (one per each 10 minutes approximately). On average, the obtained detection rate is $97.43 \%$. In addition, we have analysed the detection delay considering all the true positives. On average the $80 \%$ of all the detected pedestrians were detected in less than 0.33 seconds (less than 10 frames since the pedestrian is fully visible).
Table 3: Detection Results: Detection Rate (DR) and number of False Positives (\#FP).

\begin{tabular}{lccc}
\hline Id. & $\begin{array}{c}\text { Lighting } \\
\text { Conditions }\end{array}$ & DR & \# FP \\
\hline 1 & Daytime & $99,79 \%$ & 1 \\
2 & Daytime & $97,01 \%$ & 2 \\
3 & Nighttime & $97,17 \%$ & 0 \\
\hline Total & & $97,43 \%$ & 3 \\
\hline
\end{tabular}

\subsection{Pedestrian Behavioural Modelling}

All the variables collected by means of automatic and manual supervision mechanisms are used to define a probabilistic model able to reasoning about the pedestrian intent (walk or wait?). Although a total of 259 pedestrian intent were collected, the data was unbalanced since only 17 rejected gaps were collected. In addition, a total of 114 accepted gaps corresponded to groups of more than 2 pedestrians and no one rejected gap was collected in these cases. In order to alleviate these effects, we did not take into account data corresponding to groups of pedestrians of more than 2 people. Accordingly a total of $17 / 128 \mathrm{rejected} /$ accepted gaps were collected. We firstly estimate the distribution of accepted gaps in terms of time-to-collision. A log-normal distribution is fitted to the data (see Figure 8). The obtained results are in concordance with previous studies (Koh and Wong, 2014).

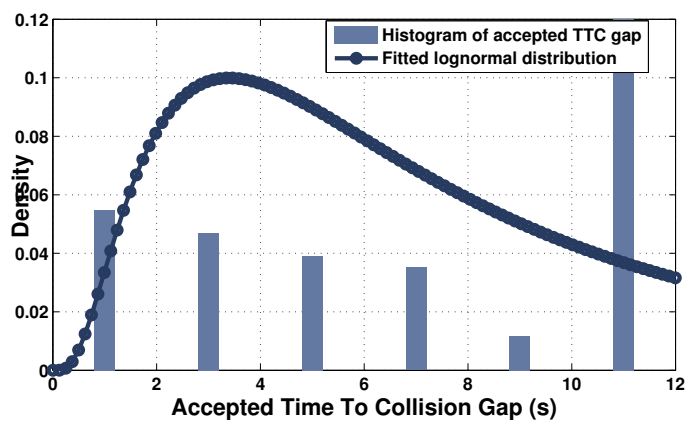

Figure 8: Accepted gap distribution fitted by a lognormal distribution.

In order to model the decision to accept the gap a binary logistic regression was used. World state $w \in\{0,1\}$ is modeled following a Bernouilli distribution with one parameter $\lambda$ and contingent to the data $x$. In this case the data $x$ corresponds to the variables described in Table 1. Accordingly, $\operatorname{Pr}(w=1)=\lambda$ for accepting the gap and $\operatorname{Pr}(w=0)=1-\lambda$ for rejecting the gap. A linear combination of the inputs $\beta^{T} x$ is used to model the parameter $\lambda$ using a sigmoid function to ensure that $0 \leq \lambda \leq 1$. Thus, the probability of accepting the gap contingent to the data is given by: 


$$
\operatorname{Pr}(w \mid x, \beta)=\operatorname{Bern}_{w}\left[\operatorname{sig}\left(\beta^{T} x\right)\right]=\frac{1}{1+e^{-\beta^{T} x}}
$$

As proposed by previous studies (Schmidt and Farber, 2009), (Koh and Wong, 2014), there is a strong correlation between the time-to-collision and the road-crossing probability. Accordingly, the first logistic regression was performed using the time-tocollision as the unique predictor variable. Figure 9 shows the accepted/rejected gaps depending on the time-to-collision and the logistic regression model. The time-to-collision variable made a significant contribution to the regression model $(p=.0004)$. The predictive quality of the overall model was $88.97 \%$ correct classifications.

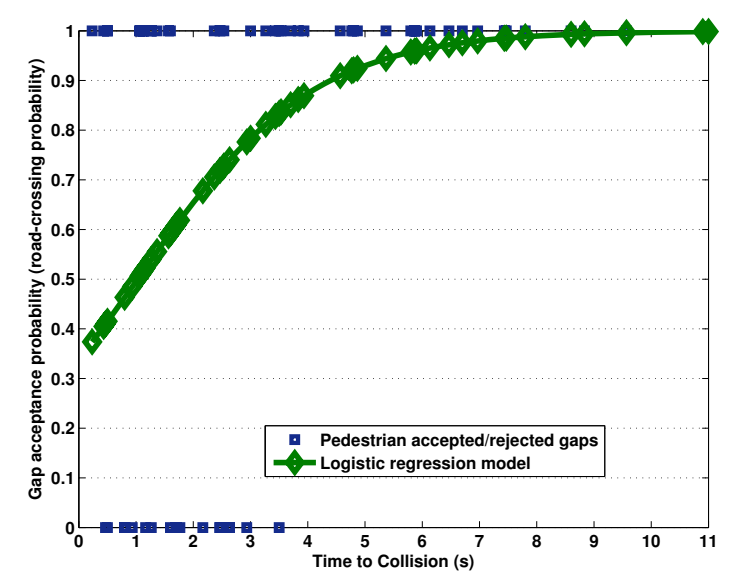

Figure 9: Accepted/rejected gaps as a function of the timeto-collision and logistic regression model obtained.

The probability of accepting gaps was further modelled using all the variables listed in Table 1. In order to analyse the significance of each variable, a sequential feature selection method was applied. Timeto-collision is selected as the first variable to enter into the model. Then, the other independent variables were put into the model one by one leading to several candidate feature subsets. A 10-fold crossvalidation is applied to each feature subset. The whole process stopped until no additional variable can enter the model. Through this process, the variables selected for the final model (see Table 4) were time-tocollision (TTC) and waiting time (WT) and the following posterior is thus given:

$$
\operatorname{Pr}(w=1 \mid x, \beta)=\frac{1}{1+e^{-0.9772-0.7833 T T C+0.6264 W T}}
$$

The predictive quality of the overall model was $93.10 \%$ correct classifications, which represents an improvement greater than $4 \%$ with respect to the model just based on time-to-collision. Variables such
Table 4: Sequential feature selection results.

\begin{tabular}{lcccc}
\hline Variable & $\beta_{i}$ & Std. & $\mathbf{t}$ & $\mathbf{p}$ \\
\hline TTC & .7833 & .2366 & 3.31 & .0009 \\
WT & -.62643 & .2094 & -2.99 & .0028 \\
\hline
\end{tabular}

as age, type of vehicle or baggage did not provide a significant contribution since the $99 \%$ of the data corresponds to middle age pedestrians, car vehicles and pedestrian not carrying baggage. Distance and vehicle speed, which have been reported as significant in other studies (Schmidt and Farber, 2009), are here not significant. This can be partially explained by the fact that both variables are integrated in the time-tocollision variable.

\section{CONCLUSIONS AND FUTURE WORK}

In this study we have presented a stereo- and infrastructure-based pedestrian detection system specifically designed to deal with infrastructurebased safety applications as well as to assess pedestrian behavioural modelling methods. Temporal density maps are generated by means of stereo reconstruction, background modelling and temporal integration. Then, objects are detected using a region growing algorithm and classified as vehicles/pedestrians or group of pedestrians depending on the motion, size and first appearance in the scene. Each pedestrian is then tracked in $3 \mathrm{D}$, allowing the estimation of his/her position in a set of manually selected regions corresponding to the pedestrian waiting areas and the crossing region. As an example of an infrastructure safety system, a blinking luminous traffic sign is switched on to warn the drivers about the presence of pedestrians in the waiting and the crossing regions. The system runs in real time, with an overall detection rate of $97.43 \%$ and one false alarm each 10 minutes, including nighttime conditions (IR illumination).

The use of the stereo-based pedestrian detection system is extended to assess pedestrian behavioural modelling. A set of variables are usually collected by fully manual supervision of surveillance data (video sequences). However, the proposed scheme allows to automatically collect some of these variables, easing this tedious procedure. In order to validate the proposed approach, the decision of a pedestrian to cross or wait is modelled using logistic regression. Although our data is unbalanced, we obtain results that are very similar to previous studies in which time-tocollision (gap) appears as the main contribution factor. A sequential feature selection method showed 
that by introducing the waiting time dedicated by a pedestrian in the waiting area as a predictor of the logistic regression model, the overall predictive quality increases a $4 \%$, leading to an accuracy of $93.10 \%$, which clearly validates the proposed methodology.

Future works will be focused on new experiments with balanced data obtained from different locations at urban environments. In addition, experimental comparisons between manual and automatic selection of several parameters will be performed to validate the proposed automatic stereo-based pedestrian behavioural parameters collection method. Finally, a more sophisticated probabilistic predictive approach will be developed and validated to increase the effectiveness of the infrastructure-based safety measurements.

\section{ACKNOWLEDGEMENTS}

This work was supported by the Spanish Ministry of Economy under Grant ONDA-FP TRA2011-27712C02-02.

\section{REFERENCES}

Álvarez, S., Llorca, D. F., and Sotelo, M. A. (2014). Hierarchical camera auto-calibration for traffic surveillance systems. Expert Systems with Applications, 41:15321542.

Álvarez, S., Sotelo, M. A., Llorca, D. F., Quintero, R., and Marcos, O. (2012). Monocular vision-based target detection on dynamic transport infrastructures. Lecture Notes in Computer Science, 6927:576-583.

Das, S., Manski, C. F., and Manuszak, M. D. (2005). Walk or wait? an empirical analysis of street crossing decisions. Journal of Applied Econometrics, 20(4):529548.

EC (2012). Basic fact sheets 2012. mobility and transport, road safety. site: http://ec.europa.eu/transport/ road_safety/specialist/statistics/index_en.htm.

Favoreel, W. (2011). Pedestrian sensing for increased traffic safety and efficiency at signalized intersections. In 8th IEEE International Conference on Advanced Video and Signal-Based Surveillance.

Gerónimo, D. and López, A. M. (2014). Vision-based Pedestrian Protection Systems for Intelligent Vehicles. Springer.

Kadali, B. R. and Vedagiri, P. (2013). Modelling pedestrian road crossing behaviour under mixed traffic condition. European Transport, 15(3).

Keller, C. G., Enzweiler, M., Rohrbach, M., D. F. Llorca, C. S., and Gavrila, D. M. (2011). The benefits of dense stereo for pedestrian detection. IEEE Transactions on Intelligent Transportation Systems, 12(4):1096-1106.
Keller, C. G. and Gavrila, D. M. (2014). Will the pedestrian cross? a study on pedestrian path prediction. IEEE Transactions on Intelligent Transportation Systems. In press.

Koh, P. P. and Wong, Y. D. (2014). Gap acceptance of violators at signalised pedestrian crossings. Accident Analysis and Prevention, 62:178-185.

Kohler, S., Goldhammer, M., Bauer, S., Zecha, S., Doll, K., Brunsmann, U., and Dietmayer, K. (2013). Stationary detection of the pedestrian's intention at intersections. IEEE Intelligent Transportation Systems Magazine, 5(4):87-99.

Llorca, D. F., Milanés, V., Parra, I., Gavilán, M., Daza, I. G., Pérez, J., and Sotelo, M. A. (2011). Autonomous pedestrian collision avoidance using a fuzzy steering controller. IEEE Transactions on Intelligent Transportation Systems, 12(2):390-401.

Llorca, D. F., Sotelo, M. A., Hellín, A. M., Gavilán, M., Daza, I. G., and Lorente, A. G. (2012). Stereo regionsof-interest selection for pedestrian protection: a survey. Transportation Research Part C, 25:226-237.

Milanes, V., Llorca, D. F., Villagrá, J., Pérez, J., Parra, I., González, C., and Sotelo, M. A. (2012). Vision-based active safety system for automatic stopping. Expert Systems with Applications, 39(12):11234-11242.

Nedevschi, S., Bota, S., and Tomiuc, C. (2009). Stereobased pedestrian detection for collision-avoidance applications. IEEE Transactions on Intelligent Transportation Systems, 10(3):380-391.

Parra, I., Llorca, D. F., Sotelo, M. A., Bergasa, L. M., de Toro, P. R., Nuevo, J., Ocana, M., and GarcíaGarrido., M. A. (2007). Combination of feature extraction methods for svm pedestrian detection. IEEE Transactions on Intelligent Transportation Systems, 8(2):292-307.

Quintero, R., Almeida, J., Llorca, D. F., and Sotelo, M. A. (2014). Pedestrian path prediction using body language traits. In IV2014, IEEE Intelligent Vehicle Symposium.

Schmidt, S. and Farber, B. (2009). Pedestrians at the kerb - recognising the action intentions of humans. Transportation Research Part F, 12(4):300-310.

Weimer, D., Kohler, S., Hellert, C., Doll, K., Brunsmann, U., and Krzikalla, R. (2011). Gpu architecture for stationary multisensor pedestrian detection at smart intersections. In IV2011, IEEE Intelligent Vehicle Symposium. 